\title{
The effect of periodontal therapy on lymphocyte blastogenesis to plaque associated microorganisms
}

\author{
D. E. Lopatin, F. N. Smith, S. A. Syed and E. C. Morrison
}

Dental Research Institute and Departments of Oral Biology and Periodontics, School of Dentistry and the Department of Microbiology-Immunology, School of Medicine, The University of Michigan, Ann Arbor, Michigan, U.S.A.

\begin{abstract}
The effect of clinical treatment on the in vitro lymphocyte blastogenic response to a panel of dental plaque-associated microorganisms, as well as non-oral antigens, was assessed longitudinally in a group of twenty-three patients during treatment for periodontitis. The patients' blastogenic response to the non-oral antigens remained constant, without significant change, throughout the three year study. Following scaling, root planning, and oral hygiene instruction (hygiene phase), an increased blastogenic response to the plaque-associated stimulants (except $B$. gingivalis) was observed. Responses measured after periodontal surgery and one year following the hygiene phase (first maintenance year) were lower than pretreatment values. Between the first and second yearly maintenance phase evaluations there was a significant increase in the blastogenic response to $A$. viscosus, $B$. gingivalis, and $F$. nucleatum. Thus, periodontal therapy initially resulted in a reduction of plaque antigen-induced lymphocyte blastogenesis. However, the responses increased again with time in the absence of deteriorating clinical parameters.
\end{abstract}

(Accepted for publication August 19, 1982)

\section{Introduction}

Host immunological response to components of the bacterial flora which comprise the dental plaque is thought to play a role in the etiology of periodontal disease (Baker et al. 1976, Ivanyi \& Lehner 1971, Ivanyi, Wilton \& Lehner, 1972). Cell-mediated and humoral/secretory mechanisms, thus activated, may lead to a variety of hypersensitivity reactions which are capable of mediating the destructive aspects of inflammatory periodontal disease. Several cross-sectional immunological studies of periodontal disease comparing the blastogenic response to dental plaque microorganisms and the clinical severity of disease suggest that a correlation may exist (Baker et al. 1976, Ivanyi \& Lehner 1971, Lehner et al. 1974, Mackler et al. 1974). However, other reports have failed to show such a close relationship (Kiger, Wright \& Creamer 1974, Smith \& Lang 1977). Problems in age-matching, health assessment, evaluation of periodontal disease severity, and experimental methodology lead to difficulties in interpreting conflicting results. While monitoring the development of periodontitis is difficult (Linde, Hamp \& Löe 1973), evaluation of clinical therapy for treatment of periodontitis may provide insight into the disease pathogenesis. In an attempt to define further the relationship between immune responses and periodontitis, we monitored lymphocyte blastogenic responses during therapy and maintenance care in a population originally diagnosed as having moderate to severe periodontitis. 


\section{Materials and Methods}

\section{Subjects}

Patient group. Twenty-three patients, ages 24 60 years, having moderate and advanced periodontal lesions were selected from patients participating in a study of reattachment and maintenance of periodontal support following surgical intervention. All subjects had some periodontal pockets extending $4.0 \mathrm{~mm}$ or more apical to the cemento-enamel junction and at least 20 treatable teeth. Patients with poor health or in need of extensive restorative dentistry were not included. Pretreatment, 4 weeks following the hygienic phase (patient education, scaling, root planing, and oral hygiene instructions), one month postsurgically, and one and two years following the hygienic phase (yearly maintenance), blood samples $(50 \mathrm{ml})$ were obtained and oral hygiene was assessed on all four tooth surfaces using the Plaque Index (Silness \& Löe 1964). Gingival health was determined using the criteria of the Periodontal Disease Index (Ramfjord 1967). The level of periodontal attachment in relation to the cemento-enamel junction (Ramfjord 1959) and pocket depth were measured using a calibrated Ml probe (Marquis Dental Manufacturing Company, Aurora, Colorado, U.S.A.) with a point diameter of $0.4 \mathrm{~mm}$. The distobuccal, buccal, mesiobuccal, lingual, and mesiolingual aspects of all teeth were scored in the maxilla and mandible. Diseased sites, classified into three categories of severity on the basis of probing depth (1-3 mm, 4-6 mm, and 7 or greater) prior to treatment were compared with their post-treatment scores.

Following the hygienic phase, three different types of periodontal surgical treatments and scaling and root planing under anesthesia were implemented in each patient (one modality per quadrant) by a periodontist. After completion of the surgical phase of therapy, patients were placed on recall for prophylaxis every three months and clinical parameters recorded annually. Student's paired method was used for statistical analyses of changes in dental plaque, gingivitis, level of attachment, and depth of pocket. The difference between mean measurements within each subject before and after each treatment phase was the variable analyzed. The null hypothesis was rejected at the 0.05 level of significance.

Control group. Fifteen subjects, ages 21 to 40, evaluated as described above, were recruited from the university community (based on clinical evaluation) to serve as a "minimal disease" control group. The average scores and ranges for the 15 control subjects were found to be: gingivitis score, $0.35(0.05-0.56)$; pocket depth, $2.22 \mathrm{~mm}(2.0-2.5)$; and loss of attachment, $0.25 \mathrm{~mm}(0.0-0.6)$. Individuals were considered "minimal disease" if the gingivitis score for any tooth did not exceed 1.0, and pocket depth did not exceed $3.0 \mathrm{~mm}$ for any site.

\section{Culture stimulants}

Recent clinical isolates of the following dental plaque-associated microorganisms were used in this study: Streptococcus sanguis MS, Actinomyces viscosus GA, Bacteroides gingivalis (Coykendall, Kaczmarek \& Slots 1980), and Fusobacterium nucleatum 19F. All bacteria were cultured in the basal anaerobic broth of Syed and Loesche (1978) with esculin eliminated and both sucrose and glucose increased to $0.5 \%$. All incubation was inside a glove box (Coy Manufacturing Co., Ann Arbor, MI) maintained at $37^{\circ} \mathrm{C}$ with an atmosphere of $85 \%$ nitrogen, $10 \%$ hydrogen, and $5 \%$ carbon dioxide. Screw-cap flasks containing $500 \mathrm{ml}$ of prereduced broth were inoculated with actively growing cells. The cultures were harvested by centrifugation at $12,000 \times \mathrm{g}$ for $30 \mathrm{~min}$. The cell pellets were extensively washed in sterile phosphate buffered saline (PBS, 0.05 $\mathrm{M} \mathrm{PO}_{4}$, $0.15 \mathrm{M} \mathrm{NaCl}, \mathrm{pH} 7.4$ ) and resuspended in sterile distilled water to give a concentration of 20 $\mathrm{mg} / \mathrm{ml}$ (wet weight). The cell harvest was approximately $100 \mathrm{mg}$ dry weight per $1000 \mathrm{ml}$ cultured medium.

The washed cells were frozen-thawed three 
times in an ethanol dry ice bath and then subjected to a total of $40 \mathrm{~min}$ of ultrasonic disruption (Heat Systems-Ultrasonics, Inc., Model W185D, 85 watts) delivered in fiveminute intervals with alternating periods of cooling in an ice bath. Cell disruption, as evaluated by gram staining and phase contrast microscopy, was greater than $95 \%$. The cell wall/whole cell mixture was centrifuged at low speed $(800 \times \mathrm{g}$ for $5 \mathrm{~min})$ followed by a high speed centrifugation of the supernatant fraction $(12,000 \times \mathrm{g}$ for $30 \mathrm{~min})$ to pellet the cell wall fragments. This was repeated six times followed by a final resuspension of the cell walls in distilled water and lyophilization.

The following non-orally associated control stimulants were obtained from commercial sources : tetanus toxoid fluid, USP (Eli Lilly and Co., Indianapolis, IN), streptolysin O (Difco Laboratories, Detroit, MI), and mumps antigen (M.A. Bioproducts, Walkersville, MD). All stimulants were aliquoted and stored at $-70^{\circ} \mathrm{C}$ until required. To ensure consistency, sufficient material of a single lot was prepared of each stimulant to preclude changing lots during the study.

\section{Lymphocyte culture}

The buffy coat layer from whole blood was centrifuged at $350 \times \mathrm{g}$ for $30 \mathrm{~min}$, diluted with sterile PBS and layered on ficoll-hypaque (Boyum 1968) (Ficoll-Paque, Pharmacia Fine Chemicals, Piscataway, NJ). The tubes were centrifuged at $500 \times \mathrm{g}$ for $30 \mathrm{~min}$ at $23^{\circ} \mathrm{C}$. The cells banded at the interface were aspirated, washed three times in PBS, resuspended to a final concentration of $2 \times 10^{6}$ cells $/ \mathrm{ml}$ in RPMI 1640 culture medium (Grand Island Biological Co., Grand Island, N.Y.), supplemented with $40 \mathrm{mM}$ HEPES buffer, $2 \mathrm{mM}$ glutamine, 50 units $/ \mathrm{ml}$ penicillin, and $50 \mu \mathrm{g} / \mathrm{ml}$ streptomycin.

Lymphocyte blastogenesis was assessed in a microtiter plate system containing $2 \times 10^{5} \mathrm{mo}-$ nonuclear leukocytes in a final volume of 0.20 ml RPMI 1640 containing $10 \%$ heat-inactivated autologous plasma. Dilutions of plaque- associated $(2.5-250 \mu \mathrm{g} / \mathrm{ml})$ and non-oralassociated $(1-25 \mu \mathrm{l} / \mathrm{ml})$ stimulants were added to triplicate wells. Preliminary studies (not shown) were performed to identify the optimum culture period resulting in maximum ${ }^{3} \mathrm{H}$ thymidine incorporation. Thus, a seven day interval was employed for all cultures. During the last 16 hours of culture, $2 \mu \mathrm{Ci}$ methyl- ${ }^{3} \mathrm{H}$ thymidine (Amersham/Searle, TRA 120, 5 $\mathrm{Ci} / \mathrm{mmol}$ ) was added to each well. All cultures were harvested with a multiple automated sample harvester (MASH II, M.A. Bioproducts) onto glass fiber filters. The filters were dried and counted in a liquid scintillation spectrometer.

\section{Data analysis}

Since the maximum blastogenic response to the same stimulant occurred at different stimulant concentrations for different individuals, statistical comparisons were performed using maximum CPM incorporated rather than at a predetermined stimulant concentration. Optimal stimulant concentrations for each individual did not vary during this study. The significance of differences in the blastogenic responses between control and patients groups and differences in these responses between treatment intervals were evaluated with the Student's $t$ test employing the MIDAS statistical programs developed by the Statistical Research Laboratory of the University of Michigan.

\section{Results}

Clinical parameters during therapy

A significant reduction in dental plaque was observed at all examinations in the patient group following the hygienic phase. A significant decrease in gingival inflammation was noted one month following the hygienic phase and at all subsequent intervals. There was a decrease in pocket depth and maintenance of level of attachment for all three leveis of severity for two years post surgical phase (Table 1). 
Table 1

Clinical evaluation of patient group at each treatment phase ${ }^{a}$

\begin{tabular}{|c|c|c|c|c|c|c|c|c|c|c|}
\hline \multirow[b]{2}{*}{ Plaque Index } & \multicolumn{2}{|c|}{$\begin{array}{l}\text { Pretreatment } \\
\text { Mean } \pm \text { S.D. }\end{array}$} & \multicolumn{2}{|c|}{$\begin{array}{c}\text { Hygienic Phase } \\
\text { Mean } \pm \text { S.D. }\end{array}$} & \multicolumn{2}{|c|}{$\begin{array}{l}\text { One-Month } \\
\text { Post Surgery } \\
\text { Mean } \pm \text { S.D. }\end{array}$} & \multicolumn{2}{|c|}{$\begin{array}{c}\text { One Year } \\
\text { Mean } \pm \text { S.D. }\end{array}$} & \multicolumn{2}{|c|}{$\begin{array}{l}\text { Two Years } \\
\text { Mean } \pm S . D .\end{array}$} \\
\hline & 1.75 & 0.46 & $0.49^{c}$ & 0.36 & $0.71^{\mathrm{C}}$ & 0.42 & $0.89^{c}$ & 0.49 & 0.83 & 0.46 \\
\hline Gingival Index & 1.80 & 0.20 & $0.79^{\mathrm{C}}$ & 0.37 & $0.71^{\mathrm{c}}$ & 0.40 & $0.83^{\mathrm{c}}$ & 0.37 & $0.79^{c}$ & 0.31 \\
\hline \multicolumn{11}{|l|}{ Probing Depths ${ }^{b}$} \\
\hline $1-3 \mathrm{~mm}$ & 2.35 & 0.20 & $2.18^{c}$ & 0.24 & $1.84^{\mathrm{c}}$ & 0.21 & $1.96^{c}$ & 0.68 & 1.99 & 0.97 \\
\hline 4-6 mm & 4.59 & 0.28 & $3.69^{c}$ & 0.45 & $2.73^{\mathrm{C}}$ & 0.42 & $2.94^{\mathrm{C}}$ & 1.02 & $2.95^{c}$ & 1.48 \\
\hline$\geq 7 \mathrm{~mm}$ & 7.33 & 0.43 & $5.31^{\mathrm{c}}$ & 0.81 & $3.40^{\mathrm{c}}$ & 0.87 & $3.52^{c}$ & 1.68 & $4.03^{c}$ & 1.52 \\
\hline \multicolumn{11}{|c|}{ Attachment Loss ${ }^{b}$} \\
\hline $1-3 \mathrm{~mm}$ & 2.13 & 0.72 & 2.16 & 0.78 & $2.34^{c}$ & 0.73 & 2.31 & 1.02 & 2.29 & 1.30 \\
\hline $4-6 \mathrm{~mm}$ & 3.27 & 0.94 & $2.96^{c}$ & 0.90 & $3.02^{\mathrm{c}}$ & 0.80 & 2.97 & 1.22 & 2.97 & 1.58 \\
\hline$\geq 7 \mathrm{~mm}$ & 5.90 & 1.02 & $5.02^{c}$ & 1.11 & $4.78^{\mathrm{C}}$ & 1.08 & $4.36^{\mathrm{c}}$ & 2.06 & 5.02 & 1.70 \\
\hline
\end{tabular}

${ }^{\text {a }}$ Mean value of 23 patients.

b Pocket depth/loss distance refers to mean values of those sites originally categorized during the pretreatment phase. All sites remained within original categories for subsequent evaluations.

${ }^{c}$ Significant difference from pretreatment $(p<0.05)$.

\section{Blastogenic responses}

Assessments of day to day variability of the blastogenic response using lymphocytes from control subjects were performed (not shown). In these studies, the blastogenic response to the control and plaque-associated stimulants was measured at weekly intervals for a period of one month in five control subjects. In addition, an assessment of the variation over two years, measured at six month intervals was performed. In both studies, while individual responses varied as much as $20 \%$ over the one month or two year evaluation, there was no significant difference in the group mean $(p>0.05)$ between each weekly or six month interval. The variance of weekly blastogenic measurements did not differ significantly from that of semi-annual measurements when using stimulants aliquoted and stored frozen at $-70^{\circ} \mathrm{C}$.

Prior to periodontal therapy, the in vitro lymphocyte blastogenic responses to the plaque-associated and control stimulant panels were assessed in the patient and control groups. As shown in Figs. 1 and 2, there was no significant difference between the mean lym- phocyte blastogenic response of the patient and control groups to any of the stimulants prior to therapy.

During treatment, the lymphocyte blastogenic responses of the patient group to the control stimulants were stable (Fig. 1). Following the hygienic phase $(\mathrm{PH})$, an apparent, but not significant $(\mathrm{p}>0.05)$ increase in the blastogenic responses to the plaqueassociated microorganisms was observed with the exception of $B$. gingivalis (Fig. 2). $B$. gingivalis was unchanged during that interval. Following the surgical (PS) and the first maintenance phase (MP1) periods, there was a general reduction of the blastogenic response to the plaque-associated stimulants compared to post hygienic phase values. With the exception of $B$. gingivalis $(\mathrm{p}<0.01)$, these decreases were not statistically significant $(\mathrm{p}>0.05)$. At the second yearly maintenance phase (MP2) recall visit, significant increases ( 20 out of 23 patients) in the blastogenic responses to $A$. viscosus (p $<0.02)$ and $F$. nucleatum $(\mathrm{p}<0.05)$ were observed when compared to the patient responses during the previous interval. Also, the re- 
sponses to $A$. viscosus (18 out of 23 patients, $\mathrm{p}$ $<0.02$ ) and $F$. nucleatum (17 out of 23 patients, $\mathrm{p}<0.05$ ) were significantly elevated when compared to the patients' pretreatment values. The blastogenic response to $B$. gingivalis dropped in 19 out of 23 patients $(\mathrm{p}<0.01)$ following the surgical phase, but returned to pretreatment levels during the second year of maintenance.

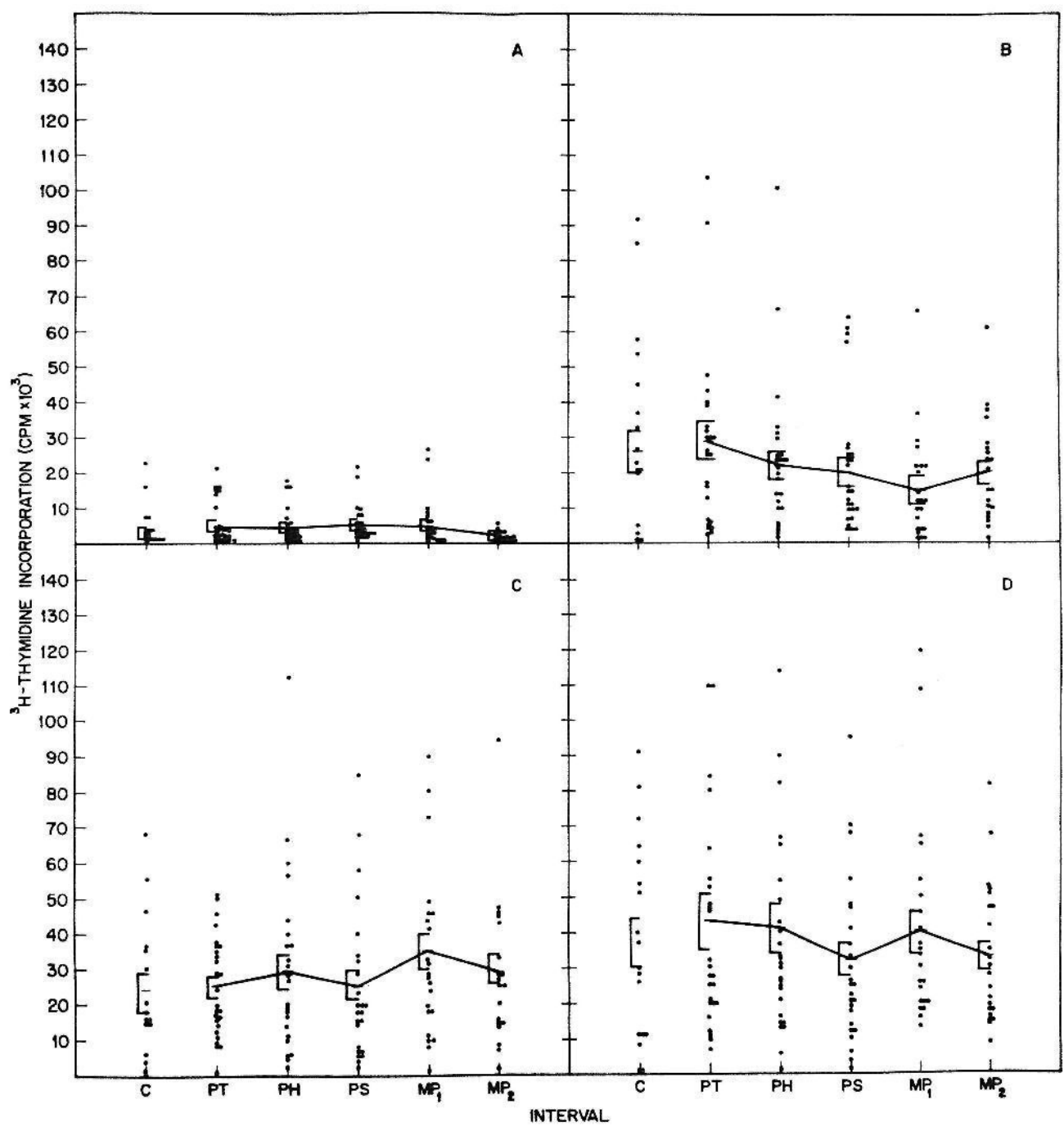

Fig. 1. Lymphocyte blastogenic responses (methyl- ${ }^{3} \mathrm{H}$-thymidine) of control subjects and periodontal patients to selected non-oral associated stimulants. CPM incorporated by unstimulated cultures (baseline) have been subtracted from those of stimulated cultures. The mean values $(+/-$ standard error of the mean) are indicated by a bar at each interval. Stimulants: baseline/unstimulated (panel A); tetanus toxoid (panel B); mumps antigen (panel C); and streptolysin $O$ (panel D). Intervals: C, control subjects; PT, pretreatment; PH, post hygienic phase; PS, post surgical phase; MP1, maintenance phase (one year post hygienic phase); MP2 maintenance phase (second year post hygienic phase). Significance levels are indicated when significant differences $(p<0.05)$ exist between indicated phase and previous phase. Significant differences between indicated phases and pretreatment phase are represented by significance levels in parentheses. 


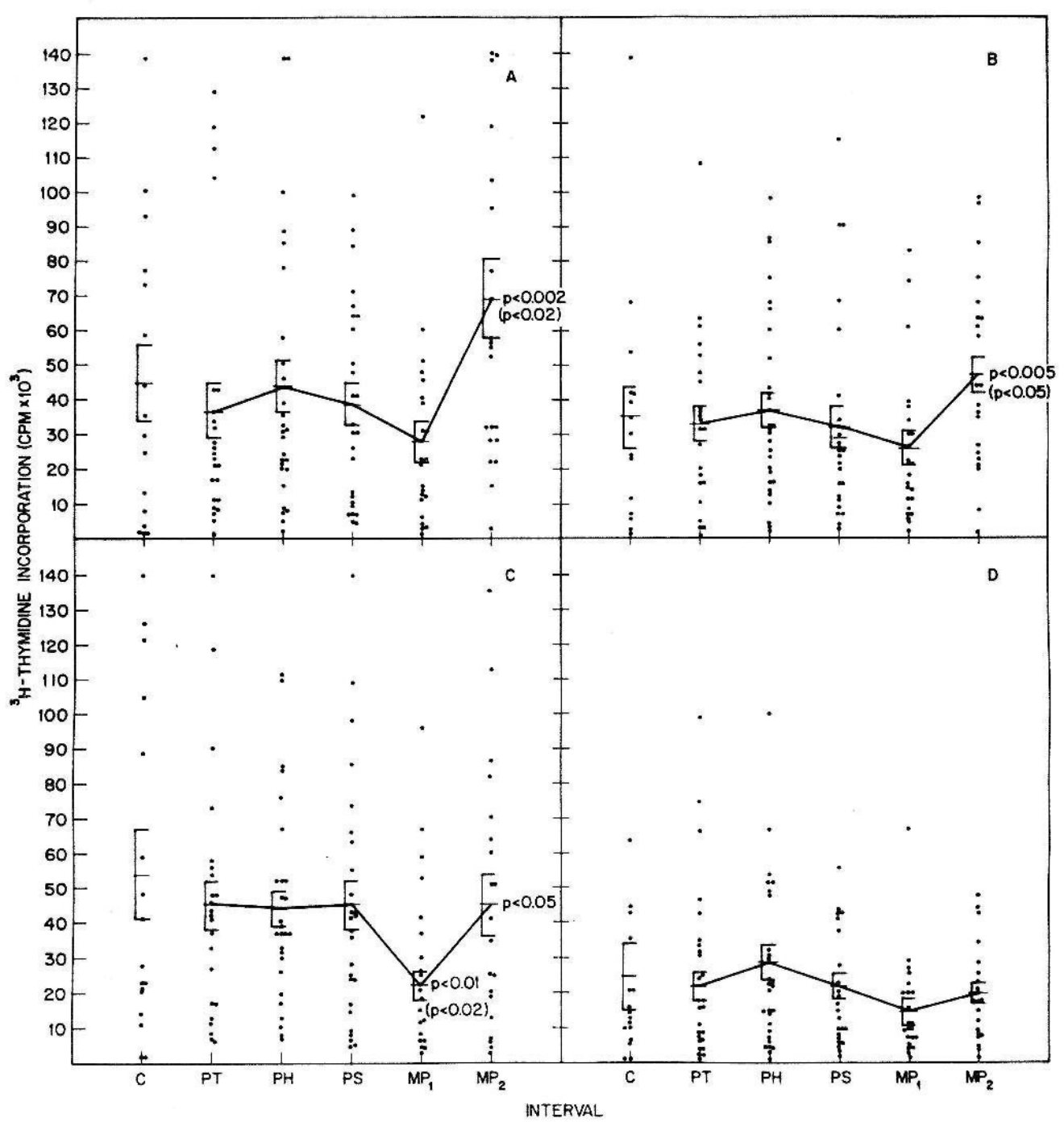

Fig. 2. Lymphocyte blastogenic responses (methyl- ${ }^{3} \mathrm{H}$-thymidine) of control subjects and periodontal patients to selected periodontal disease-associated stimulants. CPM incorportated by unstimulated cultures (baseline) have been subtracted from those of stimulated cultures. The mean values (+ + - standard error of the mean) are indicated by a bar at each interval. Stimulants: Actinomyces viscosus (panel A); Fusobacterium nucleatum (panel B); Bacteroides gingivalis (panel C); Streptococcus sanguis (panel D). Significance levels are indicated where significant differences $(p<0.05$ ) exist between indicated phase and previous phase. Significant differences between indicated phases and pretreatment phase are represented by significant levels in parentheses (See legend for Fig. 1 for description of intervals).

When compared to the original control subject responses, the patient MP2 values for $F$. nucleatum $(\mathrm{p}<0.01)$ and $A$. viscosus $(\mathrm{p}<0.05)$ were significantly elevated. Finally, there was no significant difference in the unstimulated baseline cultures, either when comparing controls and patients prior to treatment, or within the patient group during treatment. 


\section{Discussion}

A variety of in vitro correlates of cell-mediated immunity (CMI), employing solubilized dental plaque or isolated oral microorganisms as lymphocyte stimulants, have been used to implicate the immune system as an etiologic factor in periodontal disease. Such correlates have included: induction of lymphocyte transformation (Baker et al. 1976, Ivanyi \& Lehner 1971, Lang \& Smith 1977, Lehner et al. 1974, Mackler et al. 1974, Smith \& Lang 1977), lymphokine release (Horton, Oppenheim \& Mergenhagen 1973, Ivanyi et al. 1972, Mackler et al. 1974), and cellular cytotoxicity (Ivanyi et al. 1972). Histological correlation of immune parameters with periodontal disease has also been made (Clagett \& Page 1978, Genco \& Krygher 1972, Genco et al. 1974, Mackler et al. 1977, Toto, Lin \& Gargiulo 1978).

Unique mosaics of microorganisms are characteristic of the various stages and levels of severity of gingivitis and periodontitis (Löe, Theilade \& Jensen 1965, Slots 1977, Listgarten \& Hellden 1978). The microorganisms associated with increased plaque levels and gingivitis appear to be members of the genus Actinomyces, dominant in the supragingival plaque (Loesche \& Syed 1978, Smith, Lang \& Löe 1978, Syed \& Loesche 1978, Theilade et al. 1966). In longstanding gingivitis, approximately $25 \%$ of the total microbiota are found to be of the gram negative species, including Veillonella, Campylobacter, and Fusobacteria (Socransky 1970, 1977), located primarily on the surface of the plaque in subgingival sites. As the severity of periodontitis increases, subgingival plaque is characterized by increasingly higher levels of gram negative species of the genera Bacteroides, Fusobacteria, Eikenella, and Selenomonas (Slots 1977).

In a longitudinal study of the effects of treatment for severe periodontitis, lymphocytes from patients prior to treatment were specifically unresponsive (anergic) to plaque stimu- lation (Baker et al. 1978). However, following therapy (tooth extraction, scaling, and/or rigorous home care) lymphocytes from these patients became increasingly responsive to the plaque antigens, approaching the responses of the control group after six months. Patients treated with complete extractions responded identically to the patients treated by pocket elimination therapy. Thus, the increase in blastogenesis resulted from the elimination of plaque and inflammation rather than tooth loss.

The patient population in the present study was not anergic to microbial stimulation prior to treatment as were patients observed with severe periodontitis (Baker et al. 1978). In addition, no differences in blastogenesis could be detected between the control group and patients with moderate to advanced periodontitis before therapy. This finding differs from several previous reports in which blastogenic responses were higher in periodontitis patients (Baker et al. 1976, Ivanyi \& Lehner 1971, Lehner et al. 1974, Mackler et al. 1974). However, it has also been shown that individuals with apparently clinically equivalent disease (gingivitis, early or moderate periodontitis) have variable blastogenic responses (Kiger et al. 1974, Lang \& Smith 1977, Patters et al. 1976, Smith \& Lang 1977). The clinical measurements in our study showed that the control and pretreatment groups were indeed quite dissimilar.

Following $\mathrm{PH}$, an apparent (but not significant, $p>0.05$ ) increased blastogenic response to all periodontal disease-associated antigens except $B$. gingivalis was noted. Patters et al. (1977) have reported similar findings during the resolution of naturally occuring gingivitis. In their report, the blastogenic response to $A$. viscosus was elevated up to eight weeks following the initiation of therapy. In the present studies with the exception of $B$. gingivalis, to which there was a significant ( $p$ $<0.01$ at MP1) reduction of the blastogenic response, continued periodontal therapy had no 
statistically significant effect on the lymphocyte responses to plaque associated stimulants through MP1. However, the mean values during this period reflected a generally steady decrease. During the resolution of naturally occurring gingivitis, a period of increased blastogenic activity was also followed by a return to pretreatment levels (Patters et al. 1977).

At MP2, in the apparent absence of periodontal health deterioration, there was a significant increase in the blastogenic response to $A$. viscosus and $F$. nucleatum compared to the previous interval (MP1) as well as to the pretreatment measurement (PT). A significant increase in the response to $B$. gingivalis also occurred between MP1 and MP2, representing a return to pretreatment levels, rather than a hyper-responsiveness.

The apparent discrepancy of enhanced blastogenic responses to $F$. nucleatum and $A$. viscosus without deterioration of clinical signs is reminiscent of the report by Baker et al., (Baker et al. 1978) in which patients with severe periodontitis became responsive to dental plaque stimulants following therapy. However, our patients were not anergic to the stimulants and their responses were, in fact, indistinguishable from controls. With extensive maintenance therapy to prevent plaque accumulation the patients became hyperresponsive to specific stimulants. These findings complement those of Baker et al. (1978) and support a hypothesis linking periodontitis and the immunoregulatory qualities of dental plaque. The nature of the immunoregulatory control of the response to, and by, these stimulants is unclear at present. However, many of these substances posses adjuvant activity (Chen et al. 1977) or are influenced by helper activity (Lopatin, Mangan \& Horner 1981), which may ultimately facilitate immune responses to normally quiescent, nonstimulatory microbial components.

Elevated responses may also reflect microbial repopulation of the gingival sulcus and root surfaces following therapy. In a separate study, bacterial analysis of treated periodontal pockets in this group of "maintained" patients revealed repopulation with members of the genera Actinomyces and Fusobacterium several months following surgery (S. A. Syed et al., unpublished). Since the post treatment microbiota does not resemble the disease associated milieu, either in quantity or quality, presence of isolated disease-associated microorganisms may not be sufficient to foster the disease process, even though they possess immunostimulatory qualities.

The biological significance of lymphocyte transformation and its relationship to periodontal disease remains to be determined. As discussed earlier, previous investigators have linked the magnitude of transformation with disease severity. However, lymphocyte transformation does not necessarily reflect effector functions of the immune system, as shown by Ivanyi et al. (1972) who demonstrated a dissociation between transformation responses induced by plaque antigens and effector functions such as lymphokine production and cytotoxicity. Thus, elevated lymphocyte transformation to these microbial stimulants is not inconsistent with healthy gingiva.

The host-flora interactions of periodontal disease are quite complex, influenced by a flora characteristic of each stage of disease and treatment. Lymphocyte blastogenic responses to dental plaque antigens may not necessarily be indicative of disease severity. An understanding of the influence of the oral flora on immunoregulatory mechanisms and concomitantly on immunological effector functions may be necessary to understand the disease process.

\section{Acknowledgements}

We wish to thank Dr. Sigurd P. Ramfjord for permitting us to utilize patients from his longitudinal study in this study and Dr. Charles J. Kowalski and Ms. Susan Lass for assistance in statistical analysis. The expert technical 
assistance of Mrs. Frances Peebles, Mr. Ralph Woods, and Mrs. Irene Horner is acknowledged. Finally, the critical examination of the manuscript by Dr. Dennis F. Mangan is appreciated. This study was supported by Public Health Service Grant DE 02731 from the National Institute of Dental Research.

\section{References}

Baker, J. J., Chan, S. P., Socransky, S. S., Oppenheim, J. J. \& Mergenhagen, S. E. 1976. Importance of actinomyces and certain gram negative anaerobic organisms in the transformation of lymphocytes with periodontal disease. Infection and Immunity 13: 1363-1368.

Baker, J. J., Wright, W. E., Chan, S. P. \& Oppenheim, J. J. 1978. Longitudinal effects of clinical therapy and the edentulous state on the transformation of lymphocytes from patients with severe periodontitis. Clinical and Experimental Immunology 34: 199-205.

Boyum, A. 1968. Separation of leucocytes from blood and bone marrow. Scandinavian Journal of Clinical and Laboratory Investigation Suppl. 21: 1-108.

Chen, P., Farrar, J. J., Oppenheim, J. J. \& Mergenhagen, S. E. 1977. Mechanism of adjuvant activity of dental plaque: In vitro activation of residual helper T-cell precursors in T-cell-deficient murine spleen cell cultures. Infection and Immunity 17: $567-571$.

Clagett, J. A. \& Page, R. C. 1978. Insoluble immune complexes and chronic periodontal diseases in man and dogs. Archives of Oral Biology 23: 153-165.

Coykendall, A. L., Kaczmarek, R. S. \& Slots, J, 1980. Genetic heterogeneity in Bacteroides asaccharolyticus (Holdeman and Moore 1970) Finegold and Barnes 1977 (Approved lists 1980) and proposal of Bacteroides gingivalis sp. now: and Bacteroides macacae (Slots and Genco) comb. nov. International Journal of Systematic Bacteriology 30 : $559-564$.

Genco, R. J. \& Krygher, R. G. 1972. Localization of immunoglobulins, immune cells, and complement in human gingiva. Journal of Periodontal Research 7: (suppl. 10) 30-31.

Genco, R. J., Mashimo, P. S., Krygher, G. \& Ellison, S. A. 1974. Antibody-mediated effects on the periodontium. Journal of Periodontology 45: 330337.

Horton, J. E., Oppenheim, J. J. \& Mergenhagen, S. E. 1973. Elaboration of lymphotoxin by cultured human peripheral blood leukocytes stimulated with dental plaque deposits. Clinical and Experimental Immunology 13: 383-393.

Ivanyi, L. \& Lehner, T. 1971. Lymphocyte transformation by sonicates of dental plaque in human periodontal disease. Archives of Oral Biology 16: $1117-1121$.

Ivanyi, L., Wilton, J. M. A. \& Lehner, T. 1972. Cellmediated immunity in periodontal disease; cytotoxicity, migration inhibition and lymphocyte transformation studies. Immunology 22: 141-145.

Kiger, R. D., Wright, W. H. \& Creamer, H. R. 1974. The significance of lymphocyte transformation responses to various microbial stimulants. Journal of Periodontology 45: 780-785.

Lang, N. P. \& Smith, F. N. 1977. Lymphocyte blastogenesis to plaque antigens in human periodontal disease. I. Populations of varying severity of disease. Journal of Periodontal Research 12: 298309.

Lehner, T., Wilton, J. M. A., Challacombe, S. J. \& Ivanyi, L. 1974. Sequential cell-mediated immune responses in experimental gingivitis in man. Clinical and Experimental Immunology 15: 481492.

Linde, J., Hamp, S. E. \& Löe, H. 1973. Experimental periodontitis in the beagle dog. Journal of Periodontal Research 8: 1-10.

Listgarten, M. A. \& Hellden, L. 1978. Relative distribution of bacteria at clinically healthy and periodontally diseased sites in humans. Journal of Clinical Periodontology 5: 115-132.

Löe, H., Theilade, E. \& Jensen, S. B. 1965. Experimental gingivitis in man. Journal of Periodontology 36: 177-187.

Loesche, W. J. \& Syed, S. A. 1978. Bacteriology of human experimental gingivitis: Effect of plaque and gingivitis score. Infection and Immunity 21: 830-839.

Lopatin, D. E., Mangan, D. F. \& Horner, I. S. 1981. Cells involved in the mitogen-induced helper function which facilitates the blastogenic response to Actinomyces viscosus. Clinical Immunology and Immunopathology 19: 394-405.

Mackler, B. G., Altman, L. C., Wahl, S., Rosenstreich, D. C., Oppenheim, J. J. \& Mergenhagen, S. E. 1974. Blastogenesis and lymphokine synthesis by $\mathrm{T}$ and $\mathrm{B}$ lymphocytes from patients with periodontal disease. Infection and Immunity 10: 844-850.

Mackler, B. F., Frostad, K. B., Robertson, P. H. \& Levy, B. M. 1977. Immunoglobulin bearing lymphocytes and plasma cells in human periodontal disease. Journal of Periodontal Research 12: 37-45.

Patters, M. R., Genco, R. J., Reed, M. J. \& Mashimo, P. A. 1976. Blastogenic response of human 
lymphocytes to oral bacterial antigens: Comparison of individuals with periodontal disease to normal and edentulous subjects. Infection and Immunity 14: 1213-1220.

Patters, M. R., Sedransk, N. \& Genco, R. J. 1977. Lymphoproliferative response during resolution and recurrence of naturally occurring gingivitis. Journal of Periodontology 48: 373-380.

Ramfjord, S. P. 1959. Indices for prevalence and incidence of periodontal disease. Journal of Periodontology 30: 51-59.

Ramfjord, S. P. 1967. The periodontal disease index (PDI). Journal of Periodontology 38: 602-610.

Silness, J. \& Löe, H. 1964. Periodontal disease in pregnancy. II. Correlation between oral hygiene and periodontal condition. Acta Odontologica Scandinavica 22: 121-135.

Slots, J. 1977. The predominant cultivable microflora of advanced periodontitis. Scandinavian Journal of Dental Research 85: 114-121.

Smith, F. N. \& Lang, N. P. 1977. Lymphocyte blastogenesis to plaque antigens in human periodontal disease. Journal of Periodontal Research 12: 310-317.

Smith, F. N., Lang, N. P. \& Löe, H. 1978. Cellmediated immune responses to plaque antigens during experimental gingivitis in man. Journal of Periodontal Research 13: 232-239.

Socransky, S. S. 1970. Relationship of bacteria to the etiology of periodontal disease. Journal of Dental Research 49: 203-222.

Socransky, S. S. 1977. Microbiology of periodontal disease-Present status and future considerations. Journal of Periodontology 48: 497-504.

Syed, S. A. \& Loesche, W. J. 1978. Bacteriology of human experimental gingivitis: Effect of plaque age. Infection and Immunity 21 : 821-829.

Theilade, E., Wright, H. W., Jensen, S. B. \& Löe, H. 1966. Experimental gingivitis in man. II. A longitudinal clinical and bacteriologic investigation. Journal of Periodontal Research 1: 1-13.

Toto, P. D., Lin, L. M. \& Gargiulo, A. W. 1978. Immunoglobulins and complement in human periodontitis. Journal of Periodontology 49: 631634.

Address:

Dental Research Institute

The University of Michigan

Ann Arbor, Michigan 48109

U.S.A. 
This document is a scanned copy of a printed document. No warranty is given about the accuracy of the copy. Users should refer to the original published version of the material. 